\title{
Susceptibility to patterns of ciprofloxacin among nalidixic acid-resistant Salmonella isolates collected in Banepa, Nepal from enteric fever patients
}

\author{
Girija Roka, ${ }^{1}$ Subash Pandaya, ${ }^{2}$ Md. Reyad-ul Ferdous, ${ }^{2,3}$ Manson Pandey, ${ }^{4}$ Naba Raj Pokhrel, ${ }^{1}$ \\ Rashmi Shrestha, ${ }^{5}$ Tamara Towshin Alam, ${ }^{2}$ Md. Mustahsan Billah, ${ }^{6,7}$ Sayed Koushik Ahamed ${ }^{8}$ \\ ${ }^{1}$ Department of Microbiology, National College, Kathmandu, Nepal; ${ }^{2}$ Department of Pharmacy, North \\ South University, Dhaka; ${ }^{3}$ Department of Pharmacy, Progati Medical Institute, Dhaka, Bangladesh; \\ ${ }^{4}$ Department of Pharmaceutical Sciences, Sardar Bhagwan Singh Post-graduate Institute of Biomedical \\ Sciences and Research, Deheradun, India; ${ }^{5}$ Scheer Memorial Hospital, Banepa, Nepal; ${ }^{6}$ Department of \\ Pharmacy, Dhaka International University, Dhaka; ${ }^{7}$ Department of Pharmacy, Noakhali Science and \\ Technology University, Noakhali; ${ }^{8}$ Department of Pharmacy, Comilla University, Comilla, Bangladesh
}

\begin{abstract}
The present study determined the susceptibility to ciprofloxacin of nalidixic acid resistant Salmonella (NARS) isolated from enteric fever
\end{abstract}

\begin{abstract}
Correspondence: Md. Mustahsan Billah, Department of Pharmacy, Dhaka International University, 1207 Dhaka, Bangladesh.

Tel: $+880-1832-164651$

E-mail: mustahsan04pharmacy@gmail.com

Key words: Enteric fever; Multi-drug resistant; Nalidixic acid-resistant Salmonella; Minimum inhibitory concentration; Ciprofloxacin.

Conflict of interest: the authors declare no potential conflict of interest.

Contributions: GR, RS, participated in experiments, study design, manuscript preparation. SP, MMB, carried out the study design, participated in experiments, manuscript preparation, and statistical analysis. MR, supervised and directed the project. MP, TTA, checked the grammatical mistakes and corrected the final manuscript. NP, SKA, participated in experiments and statistics analysis. All authors read and approved the final version of the manuscript.
\end{abstract}

Acknowledgements: the authors are grateful to all staff of Scheer Memorial Hospital and National College for their technical support and collaboration that made this work possible.

Funding: the study was supported by the Department of Microbiology, National College, Naya Bazar, Kathmandu, Nepal. Grant No: NCKN/MSC/res. sund/91204/6513.

Received for publication: 21 September 2015.

Revision received: 19 June 2016.

Accepted for publication: 15 September 2016.

(C) Copyright G. Roka et al., 2016

Licensee PAGEPress, Italy

Journal of Biological Research 2016; 89:5527

doi:10.4081/jbr.2016.5527

This article is distributed under the terms of the Creative Commons Attribution Noncommercial License (by-nc 4.0) which permits any noncommercial use, distribution, and reproduction in any medium, provided the original author(s) and source are credited. patients at Scheer Memorial Hospital, Banepa, Nepal, from June 2012 to December 2012. The antimicrobial sensitivity to nalidixic acid and ciprofloxacin was determined using modified Kirby-Bauer disc diffusion and broth dilution method according to the guidelines of the Clinical and Laboratory Standard Institute. Salmonella was isolated from 34 out of 992 (3.43\%) blood cultures collected during the study period, and 10 (29.4\%) isolates were identified as Salmonella enterica serotype Typhi, while 24 (70.6\%) were identified as Salmonella enterica serotype Paratyphi. Out of the total isolates, 31 (91.2\%) were nalidixic acid-resistant Salmonella (NARS). Among NARS, the minimum inhibitory concentration values for ciprofloxacin ranged from 0.25 to $2 \mathrm{mg} / \mathrm{L}$ and were constantly higher than those shown by the nalidixic acid-susceptible Salmonella. Therefore, in typhoid Salmonella nalidixic acid resistance may be the indicator of decreased susceptibility to ciprofloxacin.

\section{Introduction}

The term enteric fever, coined to embrace both typhoid and paratyphoid fever, has been defined as a generalized infection of the reticuloendothelial system and intestinal lymphoid tissue accompanied by sustained fever and bacteremia. ${ }^{1}$ Enteric fever is potentially life threatening systemic illness characterized by high fever and abdominal complaints. Enteric fever is caused by the human adapted pathogens Salmonella enterica serotype Typhi ( $S$. Typhi) and $S$. enterica serotype Paratyphi (S. Paratyphi A, B, C). These organisms are important causes of febrile illness in crowded and impoverished populations with inadequate sanitation that are exposed to unsafe water and food and also pose a risk to travelers who visits the country of endemicity. ${ }^{2}$

Ciprofloxacin is synthetic antimicrobial agent commonly prescribed against enteric fever belongs to second generation 4-quinolones which is also known as fluoroquinolones and acts by inhibiting bacterial Topoisomerase II (DNA gyrase) which is required for DNA supercoiling and Topoisomerase IV which is required for strand separation during cell division. ${ }^{3}$ The wide spread use of ciprofloxacin in recent years (2003-2012), several treatment failures have also been reported due to decreased susceptibility of ciprofloxacin..$^{4,5}$ Generally, a single mutation (Ser-83 to Phe or Ser-83 to Tyr) in gyr A was associated with reduced susceptibility to ciprofloxacin (MIC $0.125-1 \mathrm{mg} / \mathrm{L}$ ) and nalidixic acid resistant. ${ }^{6}$

Routinely applied disc diffusion method is not convenient to identify reduced susceptibility of ciprofloxacin among NARS. Therefore, many 
literatures suggest that Nalidixic acid resistant (NAR) may be an indicator of reduced susceptibility of ciprofloxacin. ${ }^{7}$ This reduced susceptibility (MIC $0.125-1 \mathrm{mg} / \mathrm{L}$ ) can be identified by evaluating minimum inhibitory concentration (MIC) of ciprofloxacin. ${ }^{8}$

\section{Materials and Methods}

\section{Study site and study period}

This study was conducted at Scheer Memorial Hospital, Banepa from June 2012 to December 2012.

\section{Study population and ethical approval}

The study was conducted on the populations visiting Scheer Memorial Hospital, Nepal who were clinically suspected to have enteric fever, and requested for blood culture and antibiotic susceptibility testing. A total of 992 blood samples from the patients of suspected enteric fever were included in the study. Samples with improper labeling, insufficient blood volume, and inappropriate collection and transport were rejected. This research work was carried out according to ethical guidelines approved by the Bioethics Committee of Scheer Memorial Hospital, Banepa, Nepal and all the study subjects provided their consent to participate in this study.

\section{Sample processing}

These samples were collected aseptically using a disposable syringe. The top of the culture bottle was cleaned with iodine immediately before addition of blood. $5 \mathrm{ml}$ of venous blood drawn from adults was added to $50 \mathrm{ml}$ of sterile Brain Heart Infusion (BHI) broth. For children, the ratio used was $3 \mathrm{ml}$ of blood in $30 \mathrm{ml}$ BHI. The clinical history and personal data of the patients were recorded. The BHI broth culture bottles were labeled and incubated at $37^{\circ} \mathrm{C}$.

\section{Isolation}

Incubated culture bottles were checked daily for turbidity or visual changes (i.e. gas formation, discoloration of broth). If any turbidity was seen, then preliminary Gram's staining was performed. If Gram-negative rods were seen, the broth was subcultured on MacConkey agar (MA) and blood agar (BA). If no turbidity or growth on subculture was observed for 7 days the cultures were discarded.

\section{Identification}

The bacterial isolates from BA and MA were identified by Gram's staining and standard conventional Biochemical tests. Gram-negative short rods, producing non-lactose fermenting pale yellow colonies on MA and non haemolytic mucoid colonies on BA were subcultured on nutrient agar for biochemical tests.

\section{Antimicrobial susceptibility testing}

Antimicrobial susceptibility of the isolates was performed by disc diffusion method and the MIC values were determined by broth dilution method.

\section{Statistical analysis}

All data were entered into a computerized database (Microsoft Office Excel 2010, Microsoft Corporation). Descriptive analyses were completed and variables were recorded as necessary for statistical modeling using commercially available programs (SPSS 16.0 for Windows, SPSS inc. and WHONET 5.6, WHO).

\section{Results}

A total of 34 Salmonella were isolated during the study period. Out of these, 10 were $S$. Typhi and 24 were of $S$. Paratyphi. There was an increase in the isolation rate of $S$. Paratyphi compared to $S$. Typhi (Table 1). The suspected patient age was ranged from one day to 88 years old. Out of the total 992 blood samples, 458 (46.17\%) were male and $534(53.83 \%)$ were female. The prevalence of bacteremia among male was $4.15 \%$ (19/458) and among female was 2.81\% (15/534). The difference in growth of organisms in male and female was statistically insignificant $(\mathrm{P}=0.42)$ (Table 2). All 34 isolates were sensitive in-vitro

Table 1. Frequency of Salmonella isolates among different age groups and sex.

\begin{tabular}{|c|c|c|c|c|c|c|c|}
\hline \multirow{2}{*}{$\begin{array}{l}\text { Sex of } \\
\text { Patients }\end{array}$} & \multirow{2}{*}{$\begin{array}{l}\text { Name of } \\
\text { organism }\end{array}$} & \multicolumn{5}{|c|}{ Age group of patients (years) } & \multirow[t]{2}{*}{ Total } \\
\hline & & $<15$ & $15-29$ & $30-44$ & 45-59 & $\geq 60$ & \\
\hline \multirow[t]{3}{*}{ Female } & S. Typhi & 4 & 1 & 1 & 0 & 1 & 7 \\
\hline & S. Paratyphi & 2 & 2 & 1 & 2 & 1 & 8 \\
\hline & Total & 6 & 3 & 2 & 2 & 2 & 15 \\
\hline \multirow[t]{3}{*}{ Male } & S. Typhi & 3 & 0 & 0 & 0 & 0 & 3 \\
\hline & S. Paratyphi & 6 & 3 & 3 & 2 & 2 & 16 \\
\hline & Total & 9 & 3 & 3 & 2 & 2 & 19 \\
\hline
\end{tabular}

S. Typhi, Salmonella Typhi; S. Paratyphi, Salmonella Paratyphi.

Table 2. Frequency of growth on blood culture.

\begin{tabular}{lccc} 
Growth & Male & Female & Total \\
Positive & 19 & 15 & 34 \\
Negative & 439 & 519 & 958 \\
\hline Total & 458 & 534 & 992
\end{tabular}


to ciprofloxacin. The sensitivity was checked using $5 \mu$ g ciprofloxacin discs on Mueller-Hinton agar by modified Kirby-Bauer disc diffusion method (Table 3). Among total 34 isolates of Salmonella, 31 (91.18\%) were resistant to nalidixic acid (Table 4). Out of 3 nalidixic acid sensitive isolates, 2 isolates were $S$. Paratyphi and 1 isolate was $S$. Typhi. The MIC value of ciprofloxacin among nalidixic acid sensitive Salmonella (NASS) was in the range of $(0.03125-0.0625) \mathrm{mg} / \mathrm{L}$ and among nalidixic acid resistant Salmonella (NARS) was in the range of (0.25-1) $\mathrm{mg} / \mathrm{L}$ except 2 isolates. The association between NARS and reduced susceptibility of ciprofloxacin was statistically significant $(\mathrm{P}<0.001)$ (Table 5).

\section{Discussion}

Enteric fever continues to be a major health problem in Nepal. Its treatment still remains a challenge due to the rapid emergence of antibiotic resistance in the causative strains. Low isolation rate of Salmonella (3.43\%) was found in this research. The use of antibiotics prior to blood collection may possibly explain the low isolation rate of Salmonella in Nepal, since antibiotics are frequently prescribed even for mild cases of fever. ${ }^{9,10}$ The numbers of $S$. Paratyphi (41.18\%) were comparatively higher than $S$. Typhi. Increasing prevalence of $S$. Paratyphi has been reported from several studies worldwide including Nepal. ${ }^{11}$ The proportional increase of $S$. Paratyphi infections has been attributed to changing clinical attitude to investigate mild fever cases for enteric fever, changing host susceptibility, change in virulence of the organisms, and widespread use of quinolones antibiotics against $S$. Typhi in the past decade. ${ }^{12}$ In this study, the percentage of NAR $S$. Typhi was $90 \%$ and NAR $S$. Paratyphi was $91.7 \%$. High incidence of NARS isolates, ranging from 73.3 to $94.9 \%$, was described from different studies held in Nepal., ${ }^{4,13}$ Nalidixic acid screening has been suggested to detect and predict decreased ciprofloxacin susceptibility in enteric fever causing Salmonella. ${ }^{14,15}$ In our study, all 34 NARS isolates were found to be sensitive to ciprofloxacin by routine disc diffusion tests.
This is in accordance with a regional study that found that the isolates with decreased in vivo susceptibility to ciprofloxacin appear susceptible with routine disc diffusion tests. ${ }^{16}$

\section{Conclusions}

Enteric fever still remains one of the most common clinical diseases, especially in rainy seasons in Nepal, where Salmonella Paratyphi is the most prevalent causative serovar. Inability to identify reduced susceptibility to ciprofloxacin by the standard disk diffusion techniques urges for the revision of the current guidelines. NARS screening test may be a practical surrogate to the determination of ciprofloxacin MIC value and could be used as an indicator of decreased susceptibility to ciprofloxacin in order to avoid treatment failure.

Table 3. Antimicrobial-resistant patterns of Salmonella isolates.

\begin{tabular}{lcc} 
Antibiotics used & \multicolumn{2}{c}{ Resistant isolates (\%) } \\
& S. Paratyphi $(\mathbf{n = 2 4})$ & S. Typhi $(\mathrm{n}=10)$ \\
Nalidixic acid & $22(91.67)$ & $9(90)$ \\
Ciprofloxacin & 0 & 0 \\
\hline Gentamicin & 0 & 0 \\
Ofloxacin & 0 & 0 \\
\hline Co-trimoxazole & $2(8.33)$ & $4(40)$ \\
Ampicillin & $4(16.67)$ & $5(50)$ \\
Cefixime & 0 & 0 \\
Ceftriaxone & 0 & 0 \\
Tetracycline & $2(8.33)$ & $3(30)$ \\
Chloramphenicol & $2(8.33)$ & $4(40)$
\end{tabular}

S. Paratyphi, Salmonella Paratyphi; $S$. Typhi, Salmonella Typhi.

Table 4. Nalidixic acid susceptibility pattern of Salmonella isolates.

\begin{tabular}{lcccc} 
Isolates & NARS & NASS & Total & Statistics \\
S. Typhi & 9 & 1 & 10 & \\
S. Paratyphi & 22 & 2 & 24 & P $=0.791$ \\
\cline { 1 - 3 } Total & 31 & 3 & 34 & \\
\hline
\end{tabular}

NARS, nalidixic acid-resistant Salmonella; NASS, nalidixic acid-sensitive Salmonella; S. Typhi, Salmonella Typhi; S. Paratyphi, Salmonella Paratyphi.

Table 5. Minimum inhibitory concentration of ciprofloxacin in nalidixic acid-sensitive and acid-resistant Salmonella isolates.

\begin{tabular}{|c|c|c|c|}
\hline MIC of ciprofloxacin (mg/L) & NASS isolates & NARS isolates & Statistics \\
\hline$\leq 0.03125$ & $2(66.67)$ & 0 & \multirow{8}{*}{$\mathrm{P}<0.001$} \\
\hline 0.0625 & $1(33.33)$ & 0 & \\
\hline 0.125 & 0 & 0 & \\
\hline 0.25 & 0 & $6(19.35)$ & \\
\hline 0.5 & 0 & $19(61.29)$ & \\
\hline 1 & 0 & $4(12.9)$ & \\
\hline 2 & 0 & $2(6.45)$ & \\
\hline Total & $3(100)$ & $31(100)$ & \\
\hline
\end{tabular}

MIC, minimum inhibitory concentration; NASS, nalidixic acid-sensitive Salmonella; NARS, nalidixic acid-resistant Salmonella. 


\section{References}

1. Kumar A, Singh S, Ahirwar SK, Nath G. Proteomics-based identification of plasma proteins and their association with the hostpathogen interaction in chronic typhoid carriers. Int $\mathrm{J}$ Infect Dis 2014;19:59-66.

2. Whitaker JA, Franco-Paredes C, del Rio C, Edupuganti S. Re-thinking typhoid fever vaccines: implications for travelers and people living in highly endemic areas. J Travel Med 2009;16:46-52.

3. Souza RB, Magnani M, Ferrari RG, et al. Detection of quinoloneresistance mutations in Salmonella spp. strains of epidemic and poultry origin. Braz J Microbiol 2011;42:211-5.

4. Chaudhary R, Sijapati K, Singh S. A study on nalidixic acid resistant Salmonella among the patients attending Shree Birendra Hospital. Med J Shree Birendra Hosp 2011;10:34.

5. Olufunmiola B, Rasheed A, Samuel A. Quinolone and multi-drug resistant Salmonella Typhi in Ibadan, Nigeria. Int J Trop Med 2012;7:103-7.

6. Ryan M, Dillon C, Adley C. NAR strains of Salmonella showing decreased susceptibility to fluoroquinolones in midwestern region of the Republic of Ireland due to mutation in the gyrA gene. J Clin Microbiol 2011;49:2077.

7. Srivani R, Muthu G, Suresh A, Sumathy G. Studies on antimicrobial susceptibility pattern of Salmonella isolates from Chennai, India. Int J Pharma Bio Scie 2011;2:5975-6299.

8. CLSI. Performance standards for antimicrobial susceptibility testing. Wayne, PA: Clinical and Laboratory Standards Institute; 2006.

9. Pokharel BM, Koirala J, Dahal RK, et al. Multidrug-resistant and extended-spectrum beta-lactamase (ESBL)-producing Salmonella enterica (serotypes Typhi and Paratyphi A) from blood isolates in Nepal: surveillance of resistance and a search for newer alternatives. Int Soc Infect Dis 2009;10:434-8.

10. Rahman BA, Wasfy M0, Maksoud MA, et al. Multi-drug resistance and reduced susceptibility to ciprofloxacin among Salmonella enterica serovar Typhi isolates from the Middle East and Central Asia. New Microb New Infect 2014;2:88-92.

11. Parry CM, Thuy CT, Dongol S, et al. Suitable disk antimicrobial susceptibility breakpoints defining $S$. enterica serovar typhi isolates with reduced susceptibility to fluoroquinolones. Agents Chemother 2010;54:5201-8.

12. Sood S, Kapil A, Das B, et al. Re-emergence of chloramphenicolsensitive Salmonella typhi. Lancet 1999;353:1241-2.

13. Acharya D, Malla S, Bhatta DR, et al. Current fluoroquinolone susceptibility criteria for Salmonella need re-evaluation. Kathmandu Univ Med J 2012;37:24-9.

14. Shirakawa T, Acharya B, Kinoshita S, et al. Decreased susceptibility to fluoroquinolones and gyrA gene mutation in the Salmonella enterica serovar Typhi and Paratyphi A isolated in Katmandu, Nepal, in 2003. Diag Microb Infect Dis 2006;54:299-303.

15. Hakanen AJ, Kotilainen P, Pitkänen S, et al. Reduction in fluoroquinolone susceptibility among non-typhoidal strains of Salmonella enterica isolated from Finnish patients. J Antimicrob Chemother 2006;57:569-72.

16. Abbasi S, Imtiaz A, Usman J, et al. Evaluation of the current trend of nalidixic acid susceptibility in typhoidal Salmonellae; a marker of therapeutic failure for the fluoroquinolones. Iran J Microbiol 2011;3:80-3. 